\title{
Pathology and host immune evasion during human leptospirosis: a review
}

\begin{abstract}
Human leptospirosis is considered as one of the most widespread and potentially fatal zoonotic diseases that causes high mortality and morbidity in the endemic regions of tropical and subtropical countries. The infection can arise from direct or indirect exposure of human through contaminated environment that contains leptospires or animal reservoirs that carry leptospires. The clinical manifestations during human leptospirosis ranges from asymptomatic, mild infections to severe and life-threatening complications involving multi-organ failures with kidneys, lungs and liver severely affected. Despite much efforts have been put in to unravel the pathogenesis during human leptospirosis, it remains obscure to which extent the host factors or the pathogen itself contribute towards the pathogenesis. Host innate immunity, especially, polymorphonuclear neutrophils and complement system are involved in the first line of defense during human leptospirosis. However, pathogenic Leptospira has acquired diverse evasion strategies to evade from host immunity and establish infection in infected hosts. Hence, in this review, we focus on organs pathology during human leptospiral infection and host evasion strategies employed by Leptospira. A profound understanding on leptospiral immunity and how Leptospira subvert the immune system may provide new insights on the development of therapeutic regimens against this species in future.
\end{abstract}

Keyword: Complement system; Host immune evasion; Leptospirosis; Pathology 